\title{
Métodos para a avaliação de experiência do usuário no design de produtos
}

\author{
Methods for evaluating user experience \\ in product design
}

\author{
Carolina de Araujo Merizi ${ }^{1}$ \\ Daniela Klafke Scheibler ${ }^{2}$ \\ Fernanda Steinbruch Araujo ${ }^{3}$ \\ Lígia de Godoy 4 \\ Marcelo Gitirana Gomes Ferreira ${ }^{5}$
}




\section{Resumo}

O presente artigo apresenta uma revisão bibliográfica dos principais métodos de avaliação de UX (Experiência do Usuário), destacando sua aplicação ao Processo de Desenvolvimento de Produto - PDP. Inicialmente é trazido o conceito de UX, bem como três métodos de desenvolvimento de produtos que servirão de base ao estudo. Alguns dos principais métodos de avaliação de UX são então apresentados, destacando seus objetivos, procedimentos, vantagens e desvantagens. Por fim, apresenta-se uma discussão dos métodos, avaliando-os quanto à etapa do PDP a que cada um melhor se adequa, sendo então sistematizados em um quadro contendo as ferramentas disponíveis para cada um e o respectivo custo.

Palavras-chave: Experiência do usuário; desenvolvimento de produto; design de produto; avaliação

\section{Abstract}

This article presents a bibliographic review of the main methods for evaluating UX (User Experience), highlighting its application to the Product Development Process - PDP. Initially brought the concept of UX, as well as three methods of product development that will serve as the basis for the study. Some of the major UX evaluation methods are then presented, highlighting their objectives, procedures, advantages and disadvantages. Finally, a discussion of the methods is presented, evaluating them regarding the stage of the PDP to which each one is best suited, being then systematized in a table containing the tools available for each one and the respective cost.

Key-words: User experience; product development; product design; evaluation 


\section{Introdução}

Durante o Processo de Desenvolvimento de Produto (PDP) é importante que sejam aplicados métodos e ferramentas de avaliação que auxiliem nas tomadas de decisão para a formalização dos projetos. São eles que ajudam a garantir uma boa avaliação do produto desde suas fases iniciais de concepção, fases aquelas que mais impactam sobre a qualidade e o custo do produto, bem como sobre o seu tempo de lançamento no mercado.

A Experiência do Usuário (EU), do inglês User Experience (UX), compreende os sentimentos e percepções que resultam da interação entre um usuário e determinado produto, sistema ou serviço. No presente artigo, os três termos, produto, sistema e serviço, estarão compreendidos no termo "produto". Mensurar a experiência do usuário de um determinado produto, então, é entender como e o quê o usuário sente enquanto interage com o produto em questão.

É a partir deste contexto que o presente artigo apresenta alguns dos principais métodos de avaliação da UX, buscando, além de descrevê-los, esclarecer a melhor forma de aplicação desses, bem como as etapas do processo de desenvolvimento de produto a que cada um deles mais se adequa, de acordo com seus autores. $O$ artigo finaliza com uma discussão acerca dos métodos, elencando pontos fortes e fracos de cada um deles na sua aplicação ao PDP. Através de um quadro comparativo, apresenta as ferramentas disponíveis para aplicação dos métodos, bem como em qual fase do PDP cada um deles é mais adequado.

\section{Referencial Teórico}

\subsection{Experiência do Usuário}

Diariamente, as pessoas tornam-se "usuárias" das coisas que as cercam. Isso acontece ao sacar dinheiro no caixa eletrônico, entrar no ônibus, lavar a louça, escovar os dentes, ou seja, ao interagir com - usar - objetos e produtos projetados para executar uma determinada função. Essas interações provocam experiências que podem ser interpretadas como boas ou más experiências pelo usuário (TEIXEIRA, 2014). O termo Experiência do Usuário tem origem na tradução do inglês "User Experience", comumente abreviado na sigla UX. A expressão diz respeito às percepções e sentimentos que uma pessoa apresenta ao interagir com um serviço, sistema ou produto.

Segundo a ISO FDIS 9241-210 (2010), pode-se definir UX como as percepções e respostas de uma pessoa que resultam do uso e/ou do uso antecipado de um produto, sistema ou serviço. Já a usabilidade é definida pela ISO 9241-11 (1998) como sendo a medida pela qual um produto pode ser usado por usuários específicos para alcançar objetivos específicos com eficácia, eficiência e satisfação em um contexto específico de uso. Bevan (2009) afirma que ambas as definições, a de usabilidade (ISO 924111,1998 ) e a de experiência do usuário (ISO FDIS 9241-210, 2010), pecam por não se preocuparem declaradamente com o tempo. Tratando-se de usabilidade, não há nada sobre a capacidade de aprendizado, no caso, a mudança da usabilidade ao longo do 
tempo, e quando fala sobre experiência do usuário, a definição não considera a forma como a experiência do usuário evolui da expectativa para experiência e reflexão do usuário sobre a mesma.

Percebe-se que, nas definições trazidas tanto pela ISO 9241-11 (1998) quanto por outros autores (JOKELA, 2006; GARRET, 2003), a usabilidade é apresentada como uma característica inerente ao produto, sistema ou serviço, enquanto a UX traz um conceito mais amplo, levando em consideração a experiência propriamente dita. Preece, Rogers e Sharp (2011) afirmam que as metas de usabilidade não são suficientes para compreender a qualidade geral da experiência do usuário, reforçando a importância das metas de experiência do usuário. Os autores sustentam que, enquanto as metas de usabilidade preocupam-se com a experiência dos usuários a partir da perspectiva do produto ou sistema, as metas de experiência do usuário preocupam-se com a experiência a partir da perspectiva do próprio usuário.

Tullis e Albert (2008) reforçam a importância que deve ser destinada à UX, à medida que a tecnologia avança, de modo a oferecer produtos que, ainda que complexos tecnologicamente, sejam eficientes, atraentes e de fácil utilização. Para que isso aconteça, faz-se necessária a utilização de métricas de UX junto ao processo de desenvolvimento de produtos, métricas essas que serão coletadas, organizadas e avaliadas através de um método definido previamente.

\subsection{Processo de Design/Desenvolvimento de Produtos}

O Processo de Desenvolvimento de Produto - PDP deve ser organizado de maneira sistemática, o que possibilita também uma avaliação de cada etapa. Rozenfeld et al. (2006) definem o PDP como um conjunto de atividades que partem de necessidades do mercado, com o objetivo de chegar a um projeto de produto viável, considerando as possibilidades e restrições tecnológicas. Os autores dividem o PDP entre três macrofases:

- Pré-Desenvolvimento: planejamento do projeto, de acordo com o planejamento estratégico alinhado com a corporação;

- Desenvolvimento: Projeto Informacional; Projeto Conceitual; Projeto Detalhado; Preparação para Produção; Lançamento do Produto;

- Pós-Desenvolvimento: acompanhamento do produto após seu lançamento no mercado, possibilitando uma avaliação de todo o seu ciclo de vida.

$\mathrm{Na}$ fase de Desenvolvimento, o Projeto Informacional compreende o conjunto de informações sobre o usuário e requisitos para o projeto. Já o Projeto Conceitual é a etapa onde serão geradas e avaliadas soluções de projetos, até que uma delas seja escolhida e no Projeto Detalhado é realizado o detalhamento das especificações do projeto, bem como protótipos para avaliação do produto. Em seguida, na etapa de Preparação para a Produção, o produto é certificado, com base nos resultados dos lotes piloto, sendo liberada a sua produção para, então, ocorrer a fase de Lançamento do Produto. 


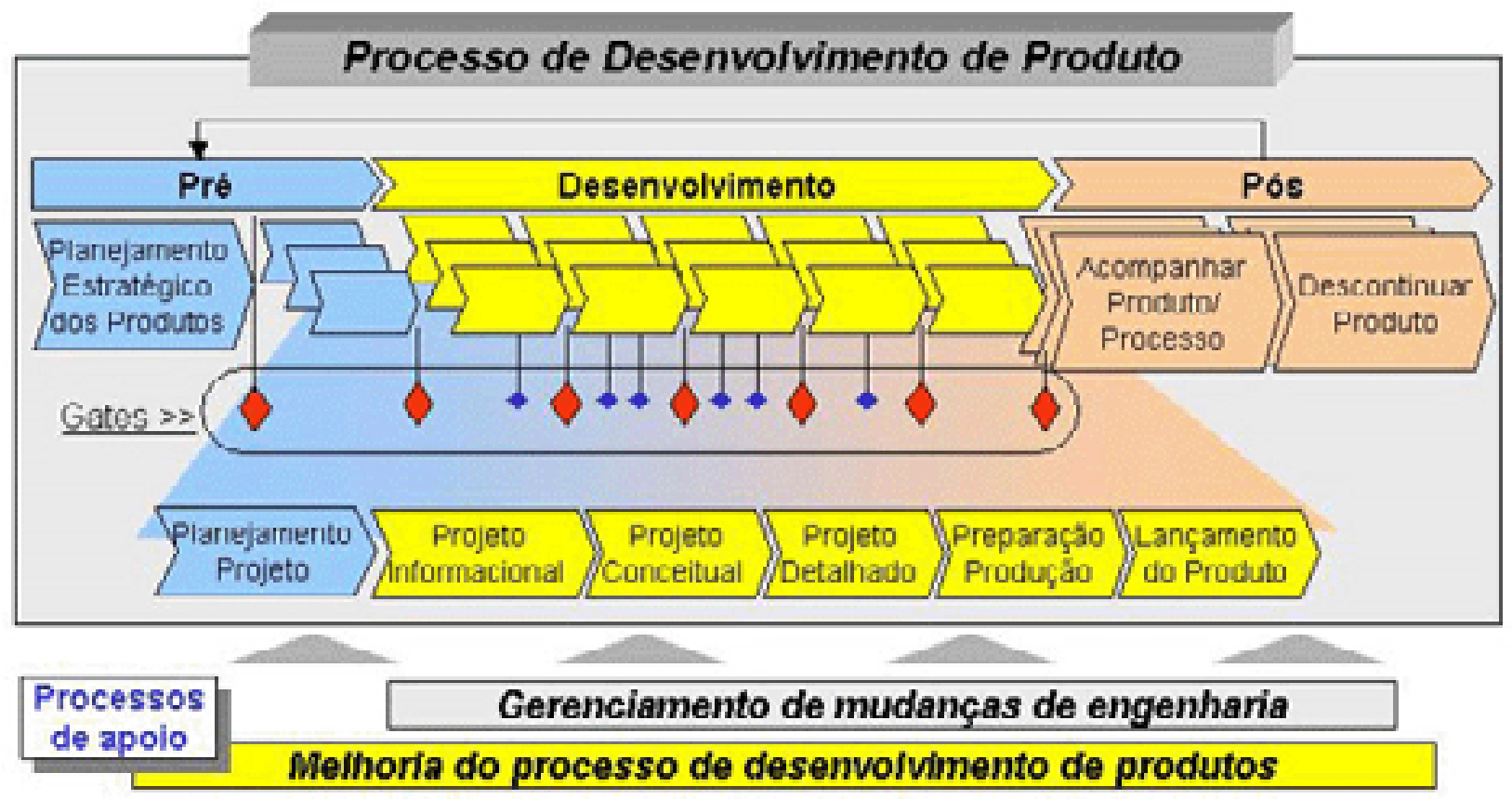

Figura 1: Representação gráfica do método de Rozenfeld et al. (2006).

Fonte: PDPNET. PDPNet. Disponível em: <http://www.pdp.org.br/>. Acesso em: 09 nov. 2017.

O Método de Desdobramento em 3 Etapas - MD3E fornece uma base conceitual para o desenvolvimento de produtos, organizado através de uma estrutura radial que permite a construção do método pelo projetista, diferenciando-se dos métodos considerados fechados, usualmente utilizados no PDP. O MD3E parte de uma etapa central, chamada de Problema de Projeto ou Necessidade Humana, desdobrando-se em três etapas básicas - Pré-concepção, Concepção e Pós-concepção - a partir das quais são indicados os desdobramentos mínimos, passando pelas atividades de Documentação e Gerenciamento do projeto, que deverão ser contempladas em todo o processo. Os desdobramentos mínimos são atividades que representam os pontos de partida para o desenvolvimento do projeto, a partir dos quais serão indicados os desdobramentos auxiliares, que poderão ter vários níveis, conforme a necessidade do projeto (SANTOS; BRUSTULIN, 2012). Os desdobramentos mínimos apresentados pelo MD3E são os seguintes:

- Pré-concepção: Planejamento do Projeto; Análise do Problema; Atributos do Produto;

- Concepção: Caminhos Criativos; Geração de Alternativas; Seleção e Adequação;

- Pós-concepção: Sub-sistemas/Componentes; Processos Produtivos; Mercado. 


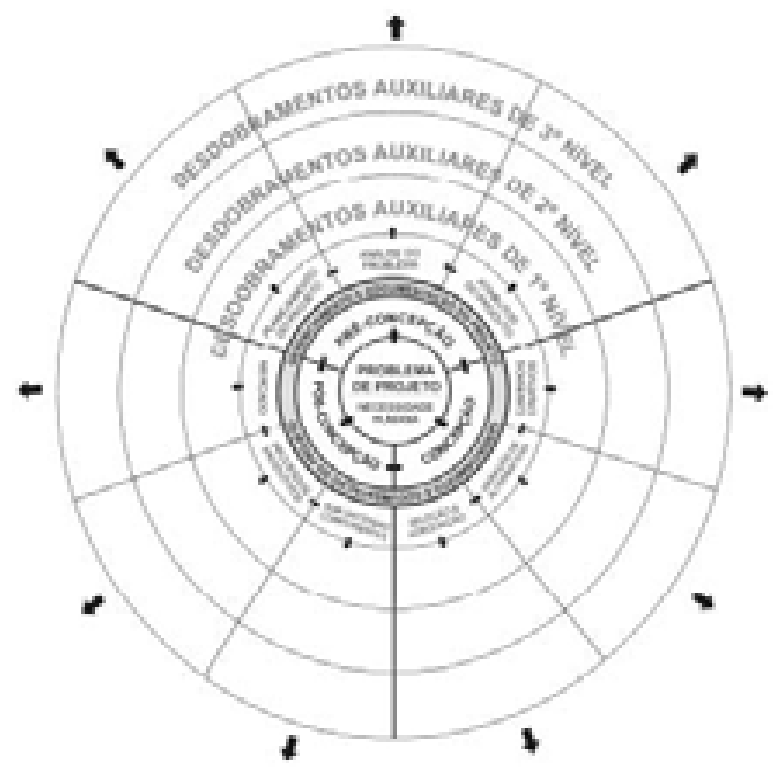

Figura 2: Representação gráfica do MD3E. Fonte: Santos (2005)

O método Guia de Orientação para o Desenvolvimento de Projetos - GODP apresenta-se, assim como o MD3E, como um modelo participativo (MERINO; GONTIJO; MERINO, 2011). O GODP traz um percurso composto por oito etapas, contidas em três fases: inspiração, ideação e implementação. É representado por um modelo cíclico, considerando a retroalimentação do processo, que traz o ser humano no centro, como conceito base (MERINO, 2016). As etapas apresentadas são as seguintes:

- Oportunidades;

- Prospecção/solicitação;

- Levantamento de dados;

- Organização e análise dos dados;

- Criação;

- Execução;

- Viabilização;

- Verificação;

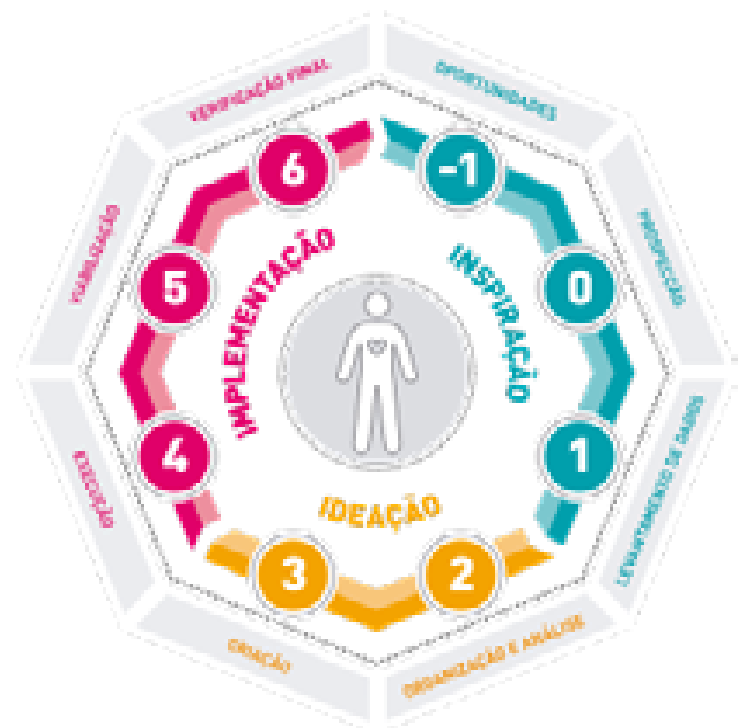

Figura 3: Representação gráfica do GODP. Fonte: Merino (2016 
Araújo (2014) destaca uma deficiência na integração entre as avaliações de usabilidade e UX e o PDP em cada uma de suas etapas, reforçando a importância dessa integração para o sucesso do produto e da sua interação com o usuário.

\section{Métodos para Avaliação da UX}

Ao longo das diversas etapas do Processo de Desenvolvimento de Produto, este costuma ser avaliado sob variados ângulos, tais como funcionais, estéticos, de uso, de fabricação, entre outros. É importante e necessário, então, que os métodos e ferramentas existentes sejam direcionados para a avaliação desses aspectos, de forma clara, buscando garantir seus resultados (BAXTER, 1995; ROZENFELD et al., 2006; BASTIEN, 2010).

Teixeira (2014) explica que as experiências dos usuários são de natureza subjetiva, pois a percepção e o pensamento são individuais e cada pessoa tem uma experiência diferente ao usar determinado produto, que pode ser influenciada por fatores humanos (habilidade, sentidos, capacidade de compreensão, humor, etc.) e por fatores externos (horário, ambiente, situação, etc.). Ainda que sejam subjetivas, essas experiências são projetadas, pensadas e desenhadas a fim de encontrar a melhor solução para os usuários. Como forma de auxiliar o desenvolvimento de projetos, existem diversos métodos complementares para avaliar a experiência do usuário de um produto. O método mais apropriado vai depender da complexidade e finalidade do produto, bem como da identificação da fase de PDP na qual a avaliação será realizada (ARAUJO, 2014). A seguir são elencados alguns dos principais métodos voltados à avaliação da experiência do usuário.

\subsection{Experience Sampling Method (ESM)}

O Experience Sampling Method (ESM) - alternativamente Experience Sampling Research, Daily Diary Method, Ecological Momentary Assessment ou ainda Ambulatory Assessment - é um método de pesquisa oriundo da Psicologia, desenvolvido por Csikszentmihalyi (1977) na Universidade de Chicago. Tem por objetivo obter auto-relatos de uma amostra representativa de momentos na vida das pessoas. De acordo com um cronograma aleatório, um dispositivo eletrônico (originalmente pagers) envia sinais para que o participante complete um questionário, no qual deve relatar a sua experiência naquele momento. O participante, na medida do possível, deve interromper a sua atividade para preencher o questionário. Usualmente, especifica-se uma faixa de horário (das 8:00 às 10:00, por exemplo) e um período de tempo (uma semana, por exemplo) ao longo do qual os relatos de experiência do participante serão coletados. Essencial é que se consiga coletar dados representativos da experiência do participante e que este receba o sinal de forma aleatória, sem aviso prévio (LARSON; CSIKSZENTMIHALYI, 2014). 


\subsection{Day Reconstruction Method (DRM)}

O Day Reconstruction Method (DRM) - Método de Reconstrução Diária - é também um método de pesquisa originário da Psicologia, desenvolvido por Kahneman et al. (2004) nos EUA. O objetivo deste método é obter memórias específicas e recentes, reduzindo assim os erros e os preconceitos de lembrança. O DRM destina-se a reproduzir as informações que seriam coletadas por experiências de sondagem em tempo real, através do ESM, por exemplo, e fornece informações de como as pessoas utilizam seu tempo e como experienciam as várias atividades e configurações de suas vidas, informações essas que não são coletadas efetivamente na amostragem de experiência (KAHNEMAN et al., 2004). Os participantes são convidados a manter uma forma de "diário do dia anterior" onde são instruídos a pensar nos seus dias em forma de sequência de episódios. Eles descrevem cada episódio respondendo perguntas sobre a situação (quando, o quê, onde, com quem) e sobre os sentimentos que eles experimentaram, usando uma tabela com doze descritores de afeto (KAHNEMAN et al., 2004). De acordo com Karapanos et al. (2009), existem variações do método. Os participantes podem, a título de exemplo, descrever três experiências mais impactantes relacionadas ao uso do produto a cada dia e, em seguida, escrever uma história que descreva em detalhes a situação, seus sentimentos e suas percepções momentâneas do produto.

\subsection{UX Curve e IScale}

O método UX Curve - método de avaliação da experiência do usuário a longo prazo - foi desenvolvido por Kujala et al. (2011) na Universidade de Tampniver, na Finlândia. O método visa auxiliar os usuários a relatar como e por que sua experiência com um produto mudou ao longo do tempo e de que forma essas razões se relacionam com a sua fidelidade como cliente. $O$ participante recebe um gráfico onde ele pode desenhar uma ou mais curvas para descrever como a sua experiência sobre um determinado produto vem evoluindo ao decorrer do tempo. A área de desenho da curva é formada por um eixo horizontal que representa o tempo e um eixo vertical, referente à intensidade da experiência. O gráfico é repartido por uma linha horizontal, que divide a área entre positiva (superior) e negativa (inferior), repartindo também o eixo vertical. Após desenhar a curva, os usuários devem descrever brevemente, por escrito ou verbalmente, o que acreditam ser as razões para as mudanças na experiência, demarcando sua localização aproximada na curva. O método pode ser utilizado para avaliar a experiência geral com o produto, ou aspectos específicos da experiência, devendo o usuário desenhar uma curva para cada aspecto avaliado, neste caso. No desenvolvimento do modelo piloto do método UX Curve foram analisados, além da experiência geral, aspectos próprios da UX, como atratividade, facilidade de uso e utilidade, bem como o grau de utilização do produto, que além de influenciar na avaliação da UX, é também influenciado pela qualidade da experiência (KUJALA et al., 2011).

O iScale foi desenvolvido por Karapanos et al. (2009) e apresentado na $\mathrm{CHI}$ 
2009 (27th International Conference on Human Factors in Computing Systems) em Boston, USA. O método tem também o objetivo de reunir dados sobre a relação do usuário com o produto durante longos períodos de tempo, buscando minimizar o viés retrospectivo, enquanto auxilia os usuários a recordarem suas experiências com o produto através de uma curva, esboçada em um gráfico de tempo versus emoção. O procedimento é muito similar ao do método UX Curve, com o diferencial de ser um sistema online. Tanto o iScale quanto o método UX Curve foram projetados para medir tendências de longo prazo em UX e entender a qualidade do projeto através da relação usuário-produto. Em princípio, ambos podem ser utilizados para medir qualquer dimensão de UX, mas as dimensões utilizadas nos estudos citados foram diferentes. Enquanto o iScale centrou-se na utilidade, facilidade de uso e inovação (KARAPANOS et al., 2010), o UX Curve centrou-se, além disso, em uma dimensão aberta geral e na atratividade (KUJALA et al., 2011).

\subsection{Product Emotion Measurement Tool (PrEmo)}

O PrEmo - Product Emotion Measurement Tool - é um instrumento criado por Desmet (2002), na Delft University of Technology, na Holanda, com o objetivo de estudar as relações entre a aparência de um produto e as reações emocionais que ele desperta no usuário. Inicialmente, o usuário é apresentado ao produto que se deseja avaliar ou a uma imagem deste. $O$ instrumento traz quatorze emoções, sete delas positivas (alegria, esperança, orgulho, surpresa agradável, satisfação, fascinação e desejo) e sete negativas (desapontamento, medo, vergonha, surpresa desagradável, insatisfação, tédio e repugnância). Tal como mostrado na Figura 4, essas emoções são representadas por imagens, para que o usuário analise o quanto a imagem do produto despertou cada uma daquelas emoções. O usuário deve selecionar uma das imagens, que apresentará uma rápida animação representativa daquela emoção, através de expressões faciais, vocais e gestuais. Após a animação, surge uma escala de cinco pontos, de 0 (emoção não sentida) a 4 (emoção fortemente sentida), para que o usuário avalie o quanto aquela emoção foi despertada pelo produto. É obrigatório que o usuário avalie cada uma das quatorze emoções, podendo ele, porém, avaliá-las na ordem que desejar e alterar suas respostas.

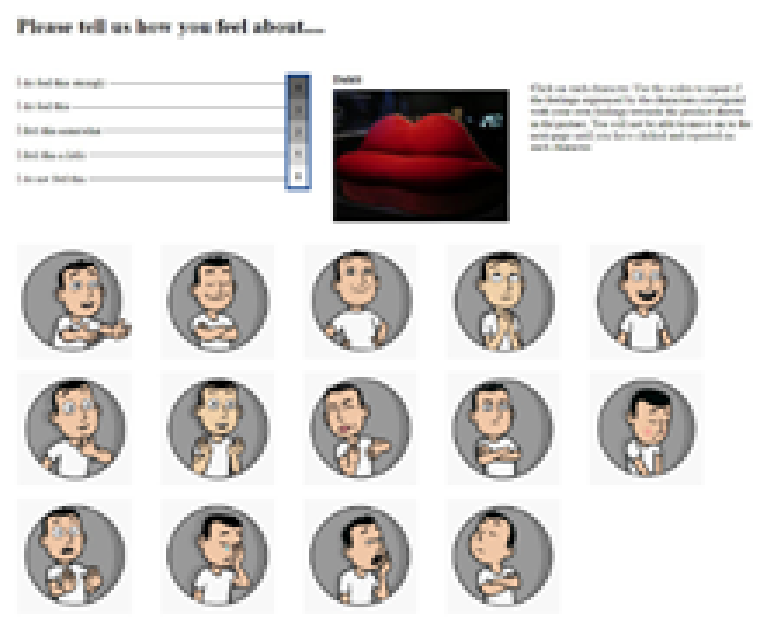

Figura 4: Captura de tela da ferramenta PrEmo. Fonte: Susagroup (2017) 

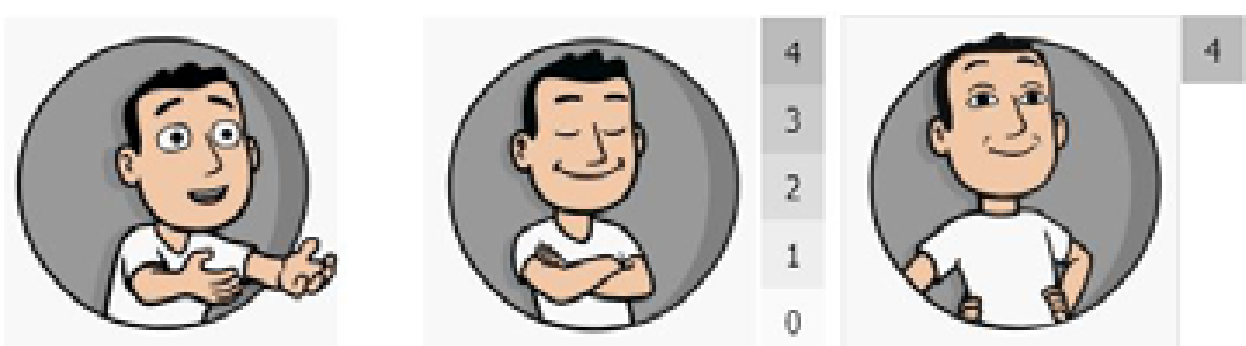

Figura 5: Sequência de ações da ferramenta PrEmo: primeiro a animação, em seguida a disposição da escala e por último a animação já avaliada. Fonte: Susagroup (2017)

Desmet (2002) reforça a importância da utilização de uma ferramenta não-verbal para avaliação das emoções, evitando barreiras à sua utilização, sejam elas culturais ou de linguagem, podendo ser aplicada nas mais diversas localidades. O autor afirma também que a ferramenta pode ser personalizada, caso o conjunto de emoções elencadas não seja adequado à avaliação de um determinado produto (DESMET, 2002).

\subsection{Change Oriented analysis of the Relationship between Product and USer (CORPUS)}

O método CORPUS - Change Oriented Analysis of the Relationship between Product and User - foi originalmente desenvolvido na Alemanha por Von Wilamowitz-Moellendorff et al. (2006). É um método baseado em entrevistas retrospectivas, com o objetivo de analisar as mudanças ocorridas na natureza da relação usuário-produto, em um período de um a dois anos.

A primeira parte do método, que Hassenzahl e Tractinsky (2006) chamam de parte estruturada, é voltada à identificação de mudanças na percepção do usuário em relação ao produto, de acordo com diferentes dimensões de qualidade. $O$ método apresenta cinco dimensões de qualidade, a saber: utilidade, usabilidade, estimulação, beleza e identidade de comunicação. No estudo feito por Hassenzahl e Tractinsky (2006) foi incluída uma sexta dimensão de qualidade, que os autores chamaram de avaliação global. Inicialmente, é apresentado ao usuário um gráfico com uma linha do tempo no eixo x e uma escala de 10 pontos no eixo y, que varia de "nem um pouco" até "muito". Ele é questionado sobre suas percepções em relação ao produto, de acordo com uma determinada dimensão de qualidade. O usuário deve marcar, por exemplo, quanto considera que o produto apresenta de usabilidade naquele momento, de 0 a 10. O mesmo questionamento é feito, então, em relação ao momento em que o usuário começou a utilizar o produto. Se as respostas dos dois questionamentos quanto àquela dimensão de qualidade (percepção atual e inicial) forem diferentes, devem ser avaliadas a direção e a forma dessa mudança, como "melhora acelerada" ou "deterioração constante", por exemplo. Por último, é solicitado que o participante discorra sobre as razões que provocaram essas mudanças, na forma de narrativas curtas, chamadas de "incidentes de mudança", de maneira a complementar as informações obtidas até então. 


\subsection{Cenários}

O método baseado em cenários, da forma que é descrita neste trabalho, foi apresentado por Rosson e Carroll em 2002. Trata-se de uma técnica que descreve eventos de uso de um determinado produto, imaginados por um ou mais usuários, através de narrativas.

Rosson e Carroll (2002) apontam alguns elementos que um cenário deve apresentar: configuração, ou estado de início; ator ou atores; objetivos das tarefas; planos para converter os objetivos em ações; avaliação ou interpretação mental; ações ou comportamentos; eventos, ou ações externas ao usuário. O avanço tecnológico, especialmente em termos de comunicação à distância em tempo real, possibilita que o método seja aplicado ainda que o usuário e o avaliador estejam em locais diferentes e em momentos diferentes, ou seja, de forma não simultânea.

\subsection{Expressões Faciais}

O estudo sobre expressões faciais tem sua origem no ano de 1872 por Charles Darwin e, posteriormente, Ekman e Friesen (1978) desenvolveram um método acerca do tema na University of California, em San Francisco, sendo apontados entre os criadores do método baseado nos estudos feitos por Darwin.

O método é utilizado para avaliar as emoções do indivíduo por meios das suas expressões faciais. Essas expressões faciais envolvem uma combinação de contrações e/ou relaxamentos de diversos músculos da face que se traduzem em: franzir da testa, movimentação/curvatura das sobrancelhas, tensionamento das pálpebras, abertura dos olhos, abertura/fechamento da boca, esticamento/encolhimento dos lábios, movimentação do queixo, entre outros. A face do usuário é filmada e as suas expressões faciais são comparadas a padrões previamente estabelecidos para as emoções, tais como aqueles descritos no FACS - Facial Action Coding System (EKMAN; ROSENBERG, 1997). Hoje, softwares de avaliação de expressões faciais são capazes de inferir, em tempo real, as emoções dos usuários. Algoritmos computacionais detectam padrões na imagem da face dos usuários e os relacionam às suas emoções. Ekman e Friesen (2003) identificam seis famílias de emoções universais (felicidade, tristeza, surpresa, medo, raiva e desgosto) e 33 expressões mistas, formadas pela combinação daquelas emoções. Ainda de acordo com Ekman e Friesen (2003), culturalmente, as emoções variam quanto ao que as provocam, bem como quanto ao seu controle frente a situações sociais, não variam, entretanto, quanto à sua aparência.

\subsection{AttrakDiff}

Este método, desenvolvido por Hassenzahl et al. (2003), na Alemanha, utiliza 28 pares de adjetivos opostos (diferenciais semânticos) para avaliar a experiência do usuário com um produto. Tal como mostrado na Figura 6, no AttrakDiff esses diferenciais semânticos apresentam-se agrupados em quatro categorias: (1) qualidade 
pragmática, que se relaciona à usabilidade e à clareza do modelo de interação; (2) atratividade, que diz respeito à qualidade estética geral do produto; (3) qualidade hedônica de identificação, relativa à identificação do usuário com o produto, dentro de um contexto social; e, por fim, (4) qualidade hedônica de estimulação, que avalia o quanto o produto estimula o crescimento pessoal, ou desenvolvimento, do usuário. Os usuários avaliam os diferenciais semânticos utilizando uma escala de sete pontos $(-3$ a +3$)$, na qual valores negativos correspondem a adjetivos que desfavorecem a UX e valores positivos a adjetivos que favorecem a UX. Para cada diferencial semântico calcula-se a média aritmética dos valores atribuídos pelos usuários. Em seguida, calculam-se as médias dos sete itens de cada uma das quatro categorias de diferenciais semânticos. O AttrakDiff também permite a realização de avaliações comparativas entre produtos concorrentes, ou de versões de um mesmo produto.

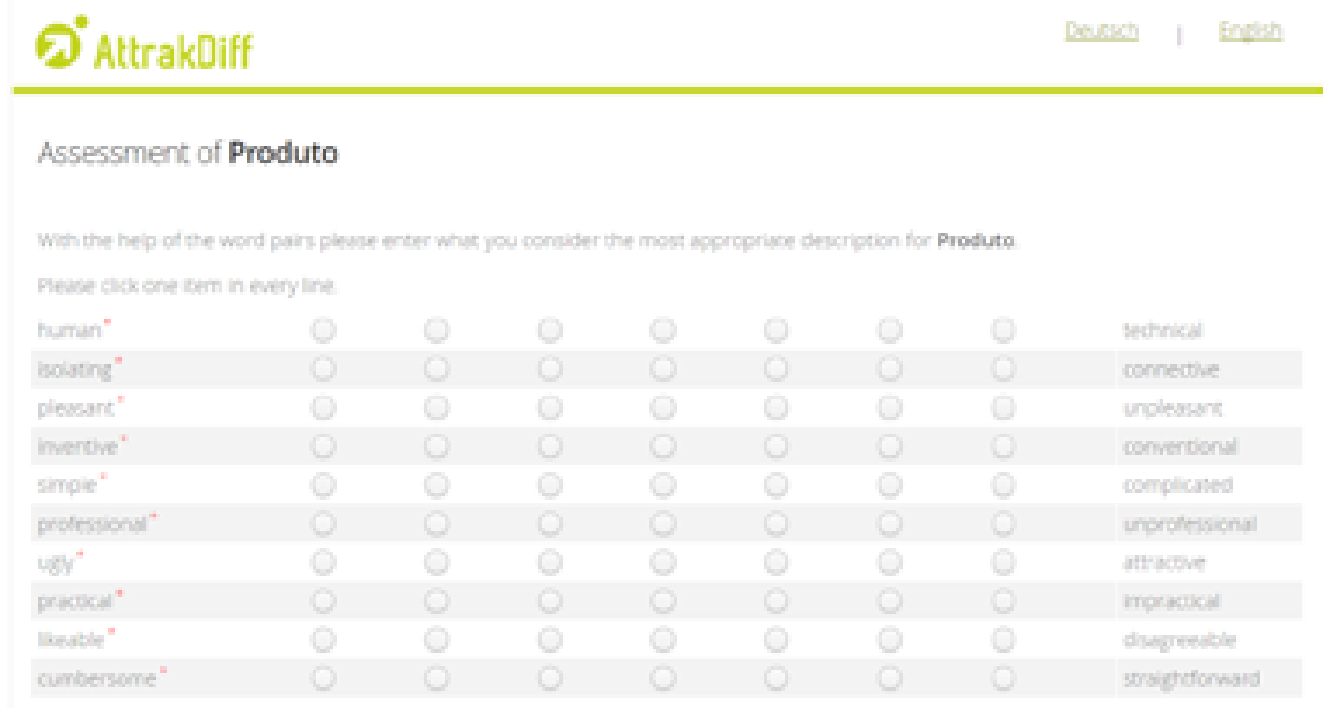

Figura 6: Captura de tela da ferramenta AttrakDiff. Fonte: UID, 2017

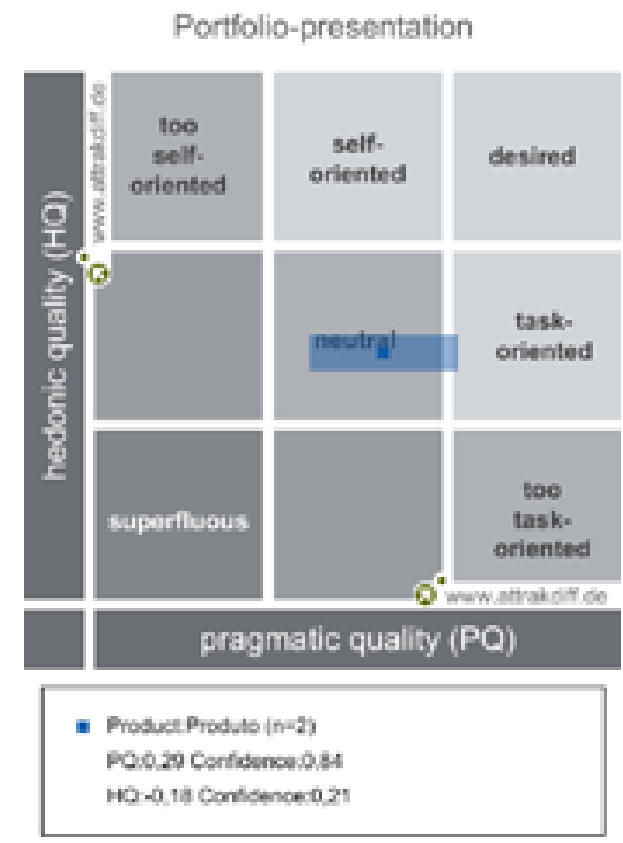

Figura 7: AttrakDiff - Instrumento de coleta de dados; portfólio de resultados; representação dos diferenciais semânticos. Fonte: UID, 2017 


\section{Discussão}

Ao escolher o método mais adequado à avaliação de experiência do usuário deve-se levar em consideração diversos fatores, como o tipo de produto que se está projetando e a fase do PDP em que será aplicada a avaliação.

Tanto o método Experience Sampling Method (ESM) quanto o Day Reconstruction Method (DRM) são oriundos da psicologia, devendo ser adaptados para a avaliação da UX com produtos e sistemas, de modo a realizá-la de forma mais eficaz. $O$ ESM provê dados mais fidedignos, uma vez que as experiências são registradas em tempo real, não necessitando o usuário relembrar experiências e fatos que ocorreram ao longo do dia. É importante, porém, que se garanta, de alguma forma, que o usuário esteja interagindo com o produto ou sistema no momento em que relata sua experiência. $O$ fato de ser realizado em tempo real pode configurar uma desvantagem do ESM, de acordo com KAHNEMAN et al. (2004), que consideram o método DRM mais eficiente, à medida que sobrecarrega menos o usuário, não interrompendo as atividades e fornecendo uma avaliação dessas ao longo do dia, não apenas em uma amostragem de momentos. Considera-se, portanto, a aplicação do DRM menos estressante para o usuário, além de trazer uma avaliação conjunta de atividades e experiências subjetivas e informações sobre a duração de cada uma, o que permite análises ponderadas por duração das experiências. O DRM apresenta também alta flexibilidade na adaptação do conteúdo dos instrumentos às necessidades do estudo específico. A aplicação do método em estudos longitudinais, buscando uma análise ao longo de um período de tempo maior (semanas ou meses, por exemplo) pode ser trabalhosa, tanto para os participantes quanto para os analistas, podendo ser desafiador recrutar e manter os participantes durante todo o estudo. Ambos os métodos, ESM e DRM, mostram-se mais adequados à avaliação de produtos prontos, portanto, seriam melhores aplicados à fase que Rozenfeld et al (2006) chamam de Pós-desenvolvimento, ao Lançamento ao Mercado (Pós-concepção) do MD3E ou à Verificação (Implementação) do GODP.

O método UX Curve, bem como o iScale, permitem que se coletem dados referentes a um longo período de tempo de uma só vez, não havendo a necessidade de comprometer os usuários durante o tempo a que se refere a análise, como ocorre com o ESM e DRM. Tanto o UX Curve como o iScale fornecem dados qualitativos sobre o que foi mais significativo e importante para os usuários, porém, o fato de dependerem da memória pode ser considerado uma desvantagem, uma vez que informações podem ser distorcidas, ou detalhes esquecidos, principalmente se coletados muito tempo depois da experiência. A aplicação de ambos os métodos demanda uma utilização frequente do produto, durante um período de tempo que possibilite a avaliação das mudanças ocorridas, sendo que o tempo indicado por Kujala et al. (2011) é entre 3 e 12 meses. Considerando a estruturação do PDP proposta por Rozenfeld et al (2006), a utilização do UX Curve e do iScale seria melhor realizada na fase de Pós-desenvolvimento, de forma a avaliar o produto final em relação ao seu uso efetivo. Em relação ao MD3E, a fase em questão corresponde ao Lançamento ao Mercado 
(Pós-concepção) e no GODP à fase de Verificação (Implementação).

O PrEmo, ao contrário dos questionários puramente verbais, utiliza-se de animações expressivas para representar as emoções dos usuários, o que facilita o acesso a emoções que são difíceis de verbalizar ou que os entrevistados estão relutantes em expressar. É difícil, porém, encontrar imagens e animações verdadeiramente inequívocas que representem a mesma emoção para a maioria das pessoas. A utilização do PrEmo restringe-se às reações emocionais ocasionadas pela aparência do produto (DESMET, 2002). Tem-se, dessa forma, uma avaliação das impressões que o usuário tem sobre aquele produto, não da experiência propriamente dita. Outra desvantagem do PrEmo é o custo, devendo ser utilizado através de um aluguel mensal. O PrEmo pode ser utilizado em todas as fases do processo de desenvolvimento de produtos. Nas fases iniciais, durante a definição do problema de projeto, levantamento de necessidades e requisitos, o PrEmo pode ser aplicado na avaliação de produtos concorrentes ou de versões anteriores do mesmo produto, caso trate-se de um redesign. Nas fases intermediárias do processo, podem ser avaliados modelos ou mockups do produto em desenvolvimento, com o objetivo de analisar as respostas emocionais a esse produto até então, possibilitando seu refinamento rumo à configuração final. Nas fases finais, a aplicação da ferramenta a protótipos funcionais busca a avaliação do produto quanto ao atendimento das necessidades delimitadas e das reações esperadas pelo usuário. Desmet (2002) acrescenta que a ferramenta demonstrou bastante utilidade quando aplicada para comunicar e tornar mais tangíveis aspectos emocionais do produto dentro da própria equipe de projeto, principalmente para profissionais de outras áreas.

O método CORPUS capta as experiências do usuário durante o uso contínuo do produto, identificando as diferentes formas com que se relaciona, além de mudanças nas percepções dos usuários ao longo do tempo de uso. No entanto Kujala et al. (2011) alertam que o método fornece apenas descrições aproximadas das tendências da experiência do usuário e algumas razões que explicam as causas de mudanças ocorridas. Em relação ao PDP, tomando como referência o método de Rozenfeld et al. (2006), o método CORPUS deve ser preferencialmente aplicado à fase de Pós-desenvolvimento, ou Lançamento ao Mercado (Pós-concepção) em relação ao MD3E, e à fase de Verificação (Implementação), em relação ao GODP.

Rosson e Carroll (2002) defendem a utilização de métodos baseados em cenários, afirmando que as descrições das pessoas utilizando o produto são essenciais para discutir e analisar como este vem modelando suas atividades. Outra vantagem apontada pelos autores é a possibilidade de utilização do método nas fases iniciais de desenvolvimento de projetos. Os cenários auxiliam os designers a responder a necessidades existentes, ao mesmo tempo que antecipam novas possíveis necessidades, de forma a minimizar as incertezas do futuro, através da formulação de um futuro desejado (MARCIAL; COSTA, 2001). As narrativas produzidas são aproveitadas de diversas formas durante o projeto, sendo utilizadas para guiar o desenvolvimento de um sistema que permitirá o uso dessas experiências (ARAUJO, 2014). Uma das possíveis desvantagens do método baseado em cenários é o risco de que as descrições fujam demasiadamente da realidade. A utilização dos métodos baseados em cenários 
no PDP se aplica melhor à fase de Projeto Informacional (Desenvolvimento) no modelo de Rozenfeld et al. (2006), à fase de Atributos do Produto (Pré-concepção) no MD3E e ao Levantamento de Dados (Inspiração) do GODP.

A utilização do métodos de expressões faciais permite a identificação e interpretação das emoções do usuário enquanto utiliza um produto, com a vantagem adicional de ser um método não invasivo. Como desvantagens, além do custo das ferramentas computacionais, pode-se apontar a possibilidade de os usuários alterarem as expressões faciais, qualificando-as, modulando-as ou falsificando-as. (EKMAN; FRIESEN, 2003). A análise de expressões faciais pode ser aplicada em todos os momentos do PDP. Sua utilização pode ser útil nas fases iniciais, para o entendimento do problema de projeto, levantamento de necessidades dos usuários e estabelecimento dos requisitos de projeto, podendo ser utilizado também para avaliar produtos concorrentes ou versões anteriores de um produto a ser reprojetado. Pode ainda ser utilizado para verificar a reação emocional do usuário a modelos do produto em desenvolvimento, como mockups. Ao final do desenvolvimento, o método pode também ser aplicado para avaliar um protótipo funcional do produto, ou o produto final, na fase de Pós-desenvolvimento (ROZENFELD et al., 2006).

O método AttrakDiff é especialmente útil para avaliar aspectos distintos da experiência do usuário com o produto, através dos diferenciais semânticos, sendo também um método de fácil utilização. O AttrakDiff mostra-se mais adequado à avaliação de produtos prontos, assim como alguns dos métodos anteriores, sendo sua aplicação ideal na fase de Pós-desenvolvimento (ROZENFELD et al., 2006), Lançamento ao Mercado (Pós-concepção) no MD3E, e Verificação (Implementação) no GODP.

O quadro a seguir elenca as principais ferramentas disponíveis para cada método e sua disponibilidade, bem como a fase do PDP a que melhor se aplica.

\begin{tabular}{|c|c|c|c|c|c|}
\hline \multirow[t]{2}{*}{ Métoda } & \multirow{2}{*}{$\begin{array}{l}\text { Ferramentas } \\
\text { Disponiveis }\end{array}$} & \multirow{2}{*}{ Custo } & \multicolumn{3}{|l|}{ Fase do PDP } \\
\hline & & & $\begin{array}{l}\text { Roxenfeld et al. } \\
(2006)\end{array}$ & $\begin{array}{l}\text { MD3E } \\
\text { (SANTOS, } \\
\text { 2005) }\end{array}$ & $\begin{array}{l}\text { GODP } \\
\text { (MERINO. } \\
\text { 2006) }\end{array}$ \\
\hline \multirow{3}{*}{$\begin{array}{l}\text { Experience } \\
\text { Sampling } \\
\text { Method } \\
\text { (ESM) }\end{array}$} & $\begin{array}{l}\text { LifeData\% } \\
\text { (UFEDATA. 2017) }\end{array}$ & US\$24SOlano & \multirow[t]{3}{*}{$\begin{array}{l}\text { Pos- } \\
\text { deserwolvimento }\end{array}$} & \multirow{3}{*}{$\begin{array}{l}\text { Lançamento ao } \\
\text { Mercado (Pús- } \\
\text { concepçao) }\end{array}$} & \multirow[t]{3}{*}{$\begin{array}{l}\text { Venthcaçao } \\
\text { (Implementecăo) }\end{array}$} \\
\hline & $\begin{array}{l}\text { MowsenseXS } \\
\text { (MOVISEN3X3, 2017) }\end{array}$ & $\begin{array}{l}500 \text { U } \\
10.000 \\
\text { credtos } \\
\text { (1 cubdito = } \\
1 \text { resposta) }\end{array}$ & & & \\
\hline & $\begin{array}{l}\text { Survey Signnal } \\
\text { (SURVEYSIGNUL, 2017) }\end{array}$ & $\begin{array}{l}\text { Us\$ } 0,10 \text { sinal } \\
\text { (1 sinal = } \\
1 \text { utilizarcan) }\end{array}$ & & & \\
\hline $\begin{array}{l}\text { Day } \\
\text { Reconstruct } \\
\text { ion Method } \\
\text { (DRM) }\end{array}$ & $\begin{array}{l}\text { Protocolo } \\
\text { (KAHANEMAN ot al., 2004) }\end{array}$ & Sem custo & $\begin{array}{l}\text { Pós- } \\
\text { desurnolvimento }\end{array}$ & $\begin{array}{l}\text { Lancamento ao } \\
\text { Morcado (Pós- } \\
\text { concepcâ) }\end{array}$ & $\begin{array}{l}\text { Verificaça final } \\
\text { (Implementaça) }\end{array}$ \\
\hline $\begin{array}{l}\text { UX Curve e } \\
\text { iScale }\end{array}$ & $\begin{array}{l}\text { Protocolos } \\
\text { (KUJALA ot al., 2011; } \\
\text { KARAPANO3 et al., 2010) }\end{array}$ & Sem custo & $\begin{array}{l}\text { Pbs: } \\
\text { deserwolvimento }\end{array}$ & $\begin{array}{l}\text { Lançamento ao } \\
\text { Mercado } \\
\text { (Pús-concepção) }\end{array}$ & $\begin{array}{l}\text { Verificacho final } \\
\text { (Implementaca) }\end{array}$ \\
\hline PrEmo & $\begin{array}{l}\text { PrEmo } \\
\text { (GUSAGROUP 2017) }\end{array}$ & 125 E/mee & Todae & Todae & Todae \\
\hline CORPUS & $\begin{array}{l}\text { Protocolo } \\
\text { (VON WILAMOWIT. } \\
\text { MOELLENDOFFF ot al; } \\
\text { 2OOS) }\end{array}$ & Sem custo & $\begin{array}{l}\text { POS- } \\
\text { dessermolvimento }\end{array}$ & $\begin{array}{l}\text { Lançamento a0 } \\
\text { Mercatbo } \\
\text { (Pbe concepçao) }\end{array}$ & $\begin{array}{l}\text { Verificação final } \\
\text { (lmplententiaģằ) }\end{array}$ \\
\hline
\end{tabular}




\begin{tabular}{|c|c|c|c|c|c|}
\hline Cenários & $\begin{array}{l}\text { Protocolo } \\
\text { (ROCEON: CARROLL. } \\
\text { 2002) }\end{array}$ & Sem custo & $\begin{array}{l}\text { Projoto } \\
\text { Informacional } \\
\text { (Deservolvimento) }\end{array}$ & $\begin{array}{l}\text { Atributos do } \\
\text { Produto } \\
\text { (Pre-conceppara) }\end{array}$ & $\begin{array}{l}\text { Levantamento de } \\
\text { Dados } \\
\text { (Inspiraça) do } \\
\text { GOOP }\end{array}$ \\
\hline \multirow[t]{5}{*}{$\begin{array}{l}\text { Expressócs } \\
\text { Facials }\end{array}$} & $\begin{array}{l}\text { Face Reader } \\
\text { (NOLDUS, 2007) }\end{array}$ & $\begin{array}{l}500<=250 \\
\text { crédtos } \\
(1 \text { credito }= \\
1 \text { minuto })\end{array}$ & \multirow[t]{5}{*}{ Todas } & \multirow[t]{5}{*}{ Todas } & \multirow[t]{5}{*}{ Todas } \\
\hline & $\begin{array}{l}\text { Affoctiva } \\
\text { (ArrCCTIVA 2017) }\end{array}$ & $\begin{array}{l}\text { US\$1/ } \\
\text { minuto }\end{array}$ & & & \\
\hline & $\begin{array}{l}\text { nVieo } \\
\text { (NMSO 2017) }\end{array}$ & Não intormado & & & \\
\hline & $\begin{array}{l}\text { Emovu } \\
\text { (EMOVu, 2017) }\end{array}$ & Não inlormedo & & & \\
\hline & $\begin{array}{l}\text { Kairos } \\
\text { (KAIROS, 2017) }\end{array}$ & $\begin{array}{l}\text { Pessoal: } \\
\text { us\$500imes } \\
\text { Corporativo: } \\
\text { us } 3 \text { inonimla }\end{array}$ & & & \\
\hline AttrakDiff & $\begin{array}{l}\text { AftrakDiff } \\
\text { (UID, 2017) }\end{array}$ & Gratuita & $\begin{array}{l}\text { Pós- } \\
\text { desemolvimento }\end{array}$ & $\begin{array}{l}\text { Lancamento ao } \\
\text { Morcado (Pos- } \\
\text { conceppốo) }\end{array}$ & $\begin{array}{l}\text { Verificação final } \\
\text { (implementaçao) }\end{array}$ \\
\hline
\end{tabular}

Quadro 1: Métodos de Avaliação de UX. Fonte: elaborado pelos autores

\section{Conclusão}

A presente pesquisa se propôs a apresentar alguns dos principais métodos de avaliação de Experiência do Usuário (UX), elencando seus pontos fortes e fracos e apontando sua adequação ao Processo de Desenvolvimento de Produto (PDP). Os métodos de avaliação de UX elencados demonstraram-se verdadeiramente úteis quando aplicados da maneira mais adequada ao PDP, reforçando a importância de se manter o ser humano ao centro do processo. O conhecimento das fases do PDP a que melhor se adequam cada um dos métodos deve guiar a sua escolha e aplicação, buscando um melhor aproveitamento das vantagens de cada um.

\section{Agradecimentos}

Os autores agradecem à Universidade do Estado de Santa Catarina (UDESC), ao Conselho Nacional de Desenvolvimento Científico e Tecnológico (CNPq), à Coordenadoria de Aperfeiçoamento de Pessoal de Nível (CAPES) e à Fundação de Apoio à Pesquisa Científica e Tecnológica do Estado de Santa Catarina (FAPESC), pelo apoio que tornou esta pesquisa possível.

\section{REFERÊNCIAS}

AFFECTIVA. Affectiva. Disponível em: <https://www.affectiva.com/>. Acesso em: 09 nov. 2017.

ARAUJO, Fernanda Steinbruch. Avaliação da experiência do usuário: uma proposta de sistematização para o processo de desenvolvimento de produtos. 2014. 238 p. Tese (Doutorado) - Universidade Federal de Santa Catarina, Centro Tecnológico, Programa de Pós-Graduação em Engenharia Mecânica, Florianópolis, 2014. Disponível em: <http://www.bu.ufsc.br/teses/PEPS5568-T.pdf> 
BASTIEN, JM Christian. Usability testing: a review of some methodological and technical aspects of the method. International journal of medical informatics, v. 79, $n$. 4, p. e18-e23, 2010.

BEVAN, Nigel. What is the difference between the purpose of usability and user experience evaluation methods. In: Proceedings of the Workshop UXEM. 2009. p. 1-4.

CSIKSZENTMIHALYI, M.; LARSON, R. Validity and reliability of the experience-sampling method. The Journal of Nervous and Mental Disease, v. 175, n. 9, p. 35-54, 2014.

DESMET, P. Designing emotions. Delft: Delft University of Technology, 2002.

EKMAN, Paul; FRIESEN, Wallace V. Unmasking the face: a guide to recognizing emotions from facial clues. Cambridge: Malor Books, 2003.

EKMAN, Paul; FRIESEN, Wallace V. Facial action coding system. 1978.

EKMAN, Paul; ROSENBERG, Erika L. What the face reveals: Basic and applied studies of spontaneous expression using the Facial Action Coding System (FACS). Oxford University Press, USA, 1997.

EMOVU. EMOVU. Disponível em: <http://emovu.com/>. Acesso em: 09 nov. 2017.

GARRETT, J. J. The Elements of User Experience: User- Centered Design for the Web. New Riders, USA, 2003.

61.

HASSENZAHL, M.; TRACTINSKY, N. User experience - A research agenda. Behaviour and Information Technology, v. 25, n. 2, p. 91-97, 2006. ISSN 0144929X

HASSENZAHL, M.; BURMESTER, M.; KOLLER, F. (2003). AttrakDiff: Ein Fragebogen zur Messung wahrgenommener hedonischer und pragmatischer Qualitat. In J. Ziegler \& G. Szwillus (Eds.), Mensch\&Computer 2003. Interaktion in Bewegung (pp. 187-196). Stuttgart, Leipzig: B. G. Teubner.

ISO FDIS 9241-210 (2009) Human-centred design process for interactive systems. ISO.

ISO 9241-11 (1998) Ergonomic requirements for office work with visual display terminals (VDTs) Part 11: Guidance on Usability. ISO. 
JOKELA, T. et al. Methods for quantitative usability require- ments: A case study on the development of the user interface of a mobile phone. Personal and Ubiquitous Computing, v. 10, n. 6, p. 345-355, 2006. ISSN 16174909 (ISSN).

104.

KAHNEMAN, Daniel et al. A survey method for characterizing daily life experience: The day reconstruction method. Science, v. 306, n. 5702, p. 1776-1780, 2004.

KARAPANOS, Evangelos et al. Measuring the dynamics of remembered experience over time. Interacting with Computers, v. 22, n. 5, p. 328-335, 2010.

KARAPANOS, E., ZIMMERMAN, J., FORLIZZI, J.B. User experience over time: an initial framework. In: CHI'09: Proceedings of the 27th International Conference on Human Factors in Computing Systems. ACM, pp. 729-738, 2009.

KAIROS. Kairos. Disponível em: <https://www.kairos.com/>. Acesso em: 09 nov. 2017.

KUJALA, Sari et al. UX Curve: a method for evaluating long-term user experience. Interacting with Computers, v. 23, n. 5, p. 473-483, 2011.

LARSON, R.; CSIKSZENTMIHALYI, M. The Experience Sampling Method. In: Flow and the Foundations of Positive Psychology. Dordrecht: Springer Netherlands, 2014. v. 15., p. 21-34.

LIFEDATA. LifeData. Disponível em: <www.lifedatacorp.com/>. Acesso em: 09 nov. 2017.

MARCIAL, Elaine Coutinho; COSTA, Alfredo José Lopes. O uso de cenários prospectivos na estratégia empresarial: vidência especulativa ou inteligência competitiva. XXV Encontro da Anpad (Enanpad), v. 25, 2001.

MERINO, Giselle Schmidt Alves Díaz. GODP - Guia de Orientação para Desenvolvimento de Projetos: Uma metodologia de Design Centrado no Usuário. Florianópolis: Ngd/ Ufsc, 2016. Disponível em: <www.ngd.ufsc.br>. Acesso em: 08 nov. 2017

MERINO, Giselle; GONTIJO, Leila Amaral; MERINO, Eugenio. O percurso do design: no ensino e na prática. In: MORAES, Dijon de; DIAS, Regina Álvares; CONSELHO, Rosemary Bom. Cadernos de Estudos Avançados em Design. Barbacena: Eduemg, 2011. p. 67-85.

MOVISENSXS. MovisensXS. Disponível em: <https://xs.movisens.com/>. Acesso em: 09 nov. 2017. 
NOLDUS. Face Reader. Disponível em: <http://www.noldus.com/human-behavior-research/products/facereader>. Acesso em: 09 nov. 2007.

NVISO. NViso. Disponível em: <http://www.nviso.ch/>. Acesso em: 09 nov. 2017.

PREECE, Jenny; ROGERS, Yvonne; SHARP, Helen. Interaction Design: Beyond Human-Computer Interaction. 3. ed. New York: John Wiley \& Sons, 2011.

ROSSON, M. E CARROLL, J. Usability Engineering: Scenario-based Development of Human-Computer Interaction. London: Academic Press, 2002.

ROZENFELD, H. et al. Gestão de desenvolvimento de produtos: uma referência para a melhoria do processo. São Paulo: Saraiva, 2006.

SANTOS, Flávio A.N.V. dos. MD3E (Método de Desdobramento em 3 Etapas): uma proposta de método aberto de projeto para uso no ensino de Design Industrial. 2005. Tese (Doutorado em Engenharia de Produção) Programa de Engenharia de Produção e Sistemas, UFSC, Florianópolis, 2005.

SANTOS, F. A. N. V. dos; BRUSTULIN, G. H. Aplicação do método aberto (md3e) em projetos ergonômicos de produtos. Human Factors in Design, v. 1, n. 1, 2012.

SUSAGROUP. Premo Tool. Disponível em: <http://www.premotool.com/>. Acesso em: 10 nov. 2017.

SURVEYSIGNAL. SurveySignal. Disponível em: <http://www.surveysignal.com/>. Acesso em: 09 nov. 2017.

TEIXEIRA, Fabricio. Introdução e boas práticas em UX design. Editora Casa do Código. Jun-2014.

TULLIS, Thomas; ALBERT, William. Measuring the User Experience: Collecting, Analyzing, and Presenting Usability Metrics. San Francisco: Morgan Kaufmann Publishers Inc., 2008.

UID. Attrakdiff. Disponível em: <http://attrakdiff.de/index-en.html>. Acesso em: 09 nov. 2017.

WILAMOWITZ-MOELLENDORFF, M.; HASSENZAHL, M.; PLATZ, A. Dynamics of user experience: how the perceived quality of mobile phones changes over time. In: User Experience - Towards a unified view, Workshop at the 4th Nordic Conference on Human-Computer Interaction. 2006. pages 74-78. 